\title{
Experimentelle Studien über den Einfluss der Anoxämie auf die Reizerregbarkeit der vegetativen und des motorischen Nerven.
}

\author{
Von \\ Ryūzō Yosomiya. \\ (四十宮龍藏) \\ (Aus der medizinischen Klinik' von Prof. Dr. T. Kato, \\ Kaiserlichen Universität zu Sendai.)
}

\section{Inhaltsverzeichnis.}

Einleitung.

Untersuchungsmethode.

Untersuchungsergebnisse.

(I) Untersuchungen bei progressiver Sauerstoff verdünnung.

(1) Verïnderungen der elektrischen Erregbarkeit des peripheren Vagus.

(2) Veränderungen der elektrischen Erregbarkeit des peripheren Sympathikus.

(3) Veränderungen der elektrischen Erregbarkeit des motorischen Nerven.

(4) Veränderungen der vegetativen Funktionen.

(II) Untersuchungen bei konstanter Sauerstoffrerdiunnung.

(1) Veränderungen der elektrischen Erregbarkeit des peripheren Vagus.

(2) Veränderungen der elektrischen Erregbarkeit des peripheren Sympathikus.

(3) Veränderungen der elektrischen Erregbarkeit des motorischen Nerven.

(4) Veränderungen der vegetativen Funktionen.

Kontrollversuche.

Zusammenfassung.

Schluss.

\section{Einleitung:}

Dass Sauerstoffmangel auf die Funktion des Atmungs- und Kreislaufzentrums oder der Zentren der vegetativen Nerven u. a. bedeutenden Einfluss ausübt, ist schon von einer grossen Anzahl von Autoren anerkannt. Von ihnen haben aber nur sehr wenige die Beeinflussung der Erregbarkeit 
der peripheren Nerven durch $\mathrm{O}_{2}$-Mangel einer eingehenden Untersuchung unterworfen. Erst in letzter Zeit fand $\mathrm{Nolf}^{1)}$ die bei Vögeln durch elektrische Reizung des peripheren Vagusstumpfes sich geltend machende Kontraktion des Muskelmagens durch $\mathrm{O}_{2}$-Verdünnung abnehmen, was er, wie er mitteilt, auf Verminderung des $p_{\mathrm{H}}$ in den sich kontrahierenden Muskelfasern zurückführt.

Ferner hat Morris ${ }^{2)}$ nachgewiesen, dass neuromyone elektrische Erregbarkeit bei durch Cyanide, Histamine oder Asphyxie herbeigeführter Anoxämie gesteigert wird und zwar umso mehr, je stärker diese wird. So kam er zum Schluss, dass diese Anoxämie der Grund sei für die Steigerung der neuromyonen elektrischen Erregbarkeit.

Aussen diesen Arbeiten liegen noch keine systematisch angestellten Versuche vor, bei welchen der Einfluss der Anoxämie auf die Erregbarkeit der vegetativen und motorischen Nerven gemessen wurde. Heute, wo die auf die Luftschifffahrt bezüglichen medizinischen Wissenschaften grosse Fortschritte gemacht haben, wären Untersuchungen wie die eben erwähnten von grossem Interesse, und zwar um so melı, als man aus den gewommenen Resultaten auf die beim Aufsteigen in grosse Höhen im Körper der Aviatiker entstehenden Veränderungen wichtige Schlüsse ziehen könnte. Das hat mich veranlasst, vorliegende Untersuchungen anzustellen.

\section{Untersuchungsmethode.}

Die erste Versuchsanordnung bestand darin, dass ich zur Ermittelung der Verïnderungen der Nervenerregbarkeit bei progressiver $\mathrm{O}_{2}$-Verdünnung, wie in meinen schon veröffentlichten Arbeiten $^{3}$, gesunde Kaninchen mittelst einer eingebundenen 'Trachealkanüle ohne Narkose mit einem Henderson'schen „Rebreather" verband, wobei sie unter progressiv abnehmender $\mathrm{O}_{2}$-Spannung atmen mussten, und während der dadurch eintretenden stetig sich vertiefenden Anoxämie viertelstündlich den zeitlichen Verlauf der Veränderungen der Reizerregbarkeit der peripheren vegetativen und des peripheren motorischen Nerven mit Induktionsströmen prüfte, die von einem du Bois-Reymond'schen Schlitteninduktorium geliefert wurden.

In der zweiten Versuchsanordnung liess ich, um die Veränderungen der Nervenerregbarkeit bei konstantem $\mathrm{O}_{2}$-Mangel zu untersuchen, gesunde

1) Nolf, Compt. rend. soc. biol., 1925, 93, $\$ 40$.

2) Morris, Brit. Journ. Exp. Path., 1922, 3, 101.

3) Yosomi y a, Tohoku Journ. Exp. Med., 1927, 8, 535. 
Tiere gleich bei Beginn des Versuches aus einem Douglas'schen Sacke, der mit normaler oder in bestimmtem Grade sauerstoffarm (ca. $7 \% \mathrm{O}_{2}$ ) gemachter Luft gefüllt war, Luft einatmen und leitete die Ausatmungsluft durch ein vollkommenes Ventil in einen anderen Gasometer ein. Zum Schluss des Versuches wurde die Gesamtsauerstoffaufnahme des Tieres im ganzen Verlaufe nach folgender Formel berechnet:

$$
\begin{aligned}
& \left(\mathrm{x}-\mathrm{x}^{\prime}\right)-\mathrm{y}=\mathrm{O}_{2} \text {-Aufnahme. } \\
& \mathrm{x}=\mathrm{O}_{2} \text {-Gehalt in dem Einatmungsluftsacke vor Beginn des Ver- } \\
& \text { suches. } \\
& \mathrm{x}^{\prime}=\mathrm{O}_{2} \text {-Gehalt in dem Einatmungsluftsacke nach Beendigung des } \\
& \text { Versuches. } \\
& \mathrm{y}=\mathrm{O}_{2^{-}} \text {-Gehalt, welcher nach Beendigung des Versuches in der } \\
& \text { Ausatmungsluftwanne noch enthalten ist. }
\end{aligned}
$$

Wenn man nach diesem Verfahren sauerstoffverdünnte Luft einatmen lïsst, so muss der Sauerstoff mangel im arteriellen Blute schneller herporgerufen werden als bei Anwendung des „Rebreathers". Früher hat Do it) nach diesem Verfahren Versuche angestellt und dadurch bestütigt, dass die Veründerungen der Gase des arteriellen Blutes 15 Minuten nach dem Einatmenlassen einer bestimmten verdïnnten Luft konstant werden und danach zeitlich keine grossen Schwankungen aufweisen. Die Veründerungen der Erregbarkeit der peripheren Nerren zu der Zeit, wo Anoxïmie konstant wurde, wurden untersucht.

Die Untersuchung des peripheren Sympathikus stellte ich an den beiderseitigen Halssympathici an. Diese Nerven wurden der Carotis entlang möglichst nahe der Clavicula durchschnitten, dann unter strenger Vorsicht nach oben in einer Länge von ca. 3-4 cm prïpariert und sehr vorsichtig und schonend das lockere Bindegewebe in der Umgebung der Nervenscheide abgelöst. Die prüparierten Nerven wurden, nachdem für Würme und Feuchtigkeit gesorgt, $\frac{1}{2}-1$ Stunde lang in situ gelassen, dann an ihnen die Reizschwelle bestimmt, welche mit dem grössten Rollenabstand eines du Bo is-Rey mond'schen Induktoriums (primüre resp. sekundïre Spirale mit 130 resp. 4300 Windungen, elektrische Quelle ein Akkumulator von 2 Volt) bezeichnet wird. Zu diesem Zwecke reizt man den Nerven an einer bestimmten, ungefïhr $1 \frac{1}{2} \mathrm{~cm}$ vom Schnittende entfernten Stelle mit einem Platinelektrodenpaar und sucht die Stromstärke (Rollenabstand), welche eben noch bemerkbare Pupillenerweiterung hervorruft. Jede einzelne Reizung dauert ca. 10 Sekunden. Die ganze Versuchsdauer hindurch wurden die Nerven genügend warm und feucht gehalten.

Auch die Untersuchung am Vagus erfolgte nach analogen Manipulationen, wie im Sympathikusversuche. Man prïpariert den Halsabschnitt des rechten Vagus sorgfiltig von der Ungebung ab und durchschneidet ibn möglichst kranialwärts in der Nähe des Ganglion jugulare und reizt, nachdem er etwa ${ }_{2}-1$ Stunde lang unter genïgender Feuchtigkeit und Wairme in situ gelassen worden ist, seinen peripheren Stumpf an ein und derselben Stelle mit einer bestimmten Stromstärke $(20 \mathrm{~cm} \mathrm{R.} \mathrm{A.)} 10$ Sekunden lang. Die dadurch zustande kommende Blutdruck- und Herzschlagabnahme werden während der ganzen Versuchsdauer viertelstündlich gemessen.

Un hierbei an beiden Vagi den Versuch anzustellen, durchschnitt ich sie beide. Da

4) Doi, Journ. Physiol., 1921, 55, 43 . 
ging aber das Versuchstier, besonders infolge Atemstörung, schon zu einer Zeit zugrunde, wo die $\mathrm{O}_{2}$-Verdünnung noch relativ schwach war, so dass unser Versuchszweck nicht erreicht werden konnte. Deshalb habe ich nur an einseitig vagotomierten Tieren Untersuchungen rorgenommen.

Zur Untersuchung des peripheren motorischen Nerven habe ich als Versuchsobjekt den linksseitigen $N$. peronaeus profundus ausgewühlt. Es wurde nümlich der linke Unterschenkel an der Aussenseite inzidiert, der Nerv durch oben erwähnte Manipulationen sorgfïltig isoliert, möglichst oben durcbschnitten und an seinem peripheren Stumpfe an ein und derselben Stelle durch eine elektrische Reizung (10 Sekunden lang) die minimale Stromstïrke gesucht, welche eine noch eben bemerkbare dorso-lateralwärts gerichtete Zehenbewegung auslösen kann.

Ferner habe ich nach denselben Methoden, vie in meinen schon veröffentlichten ${ }^{3)}$ Arbeiten, die dabei entstehenden Verïnderungen der Atmung und des Kreislaufes, der Blutgase $\left(\mathrm{O}_{2}\right.$-Linsüttigung, prozentiger $\mathrm{O}_{2}$-Sïttigung und $\mathrm{CO}_{2}$-Gehaltes des arteriellen Blutes), Grösse der Sauerstoffaufnahme, ertrïgliches $\mathrm{O}_{2}$-Verdinnungsmaximum der Einatmungsluft und Lebensdauer untersucht und ihre Beziehung zu den Erregbarkeitsverïnderungen der peripheren Nerven ermittelt.

\section{Untersuchungsergebnisse.}

(I) Veränderungen der Erregbarkeit der Nerven bei progressiver Sauerstoff ferdünnung.

Zuerst setzte ich gesunde Kaninchen durch Verbindung mit der Hendersonschen „Rebreather" progressiver $\mathrm{O}_{2}$-Verdünnung aus, und in ihrem Verlaufe wurden viertelstündlich die Veründerungen der elektrischen Erregbarkeit des peripheren Vagus, Sympathikus und motorischen Nerven nach schon geschildertem Verfahren untersucht. Im Folgenden werden die gewonnenen Ergebnisse der Reihe nach beschrieben :

(1) Veränderungen der elektrischen Erregbarkeit des peripheren Vagus.

Am zervikalen Vagusstamm wurden 5 Versuche angestellt, welche alle fast dieselben Verïnderungen ergaben.

Die Blutdruck- resp. Herzschlagverminderung durch zehnsekündliche faradische Reizung bei $20 \mathrm{~cm} \mathrm{R}$. A. betrug zuerst in normaler Luft im Durchschnitt $8 \%$ resp. $12 \%$, fing bei durchschnittlich $8 \% \mathrm{O}_{2}$ allmählich stärker zu werden an und erreichte bei ca. $6 \% \mathrm{O}_{2}$ ihr Maximum, wobei im Mittel die Blutdrucksenkung 22\% (eine Zunahme von $175 \%$ ) und die Herzverlangsamung $60 \%$ (eine Zunahme von $400 \%$ ) zühlte. Diese rührt daher, dass zu dieser Zeit die Erregbarkeit des peripheren Vagus maximal geworden ist. Danach wurde letztere mit herannahendem Endstadium 


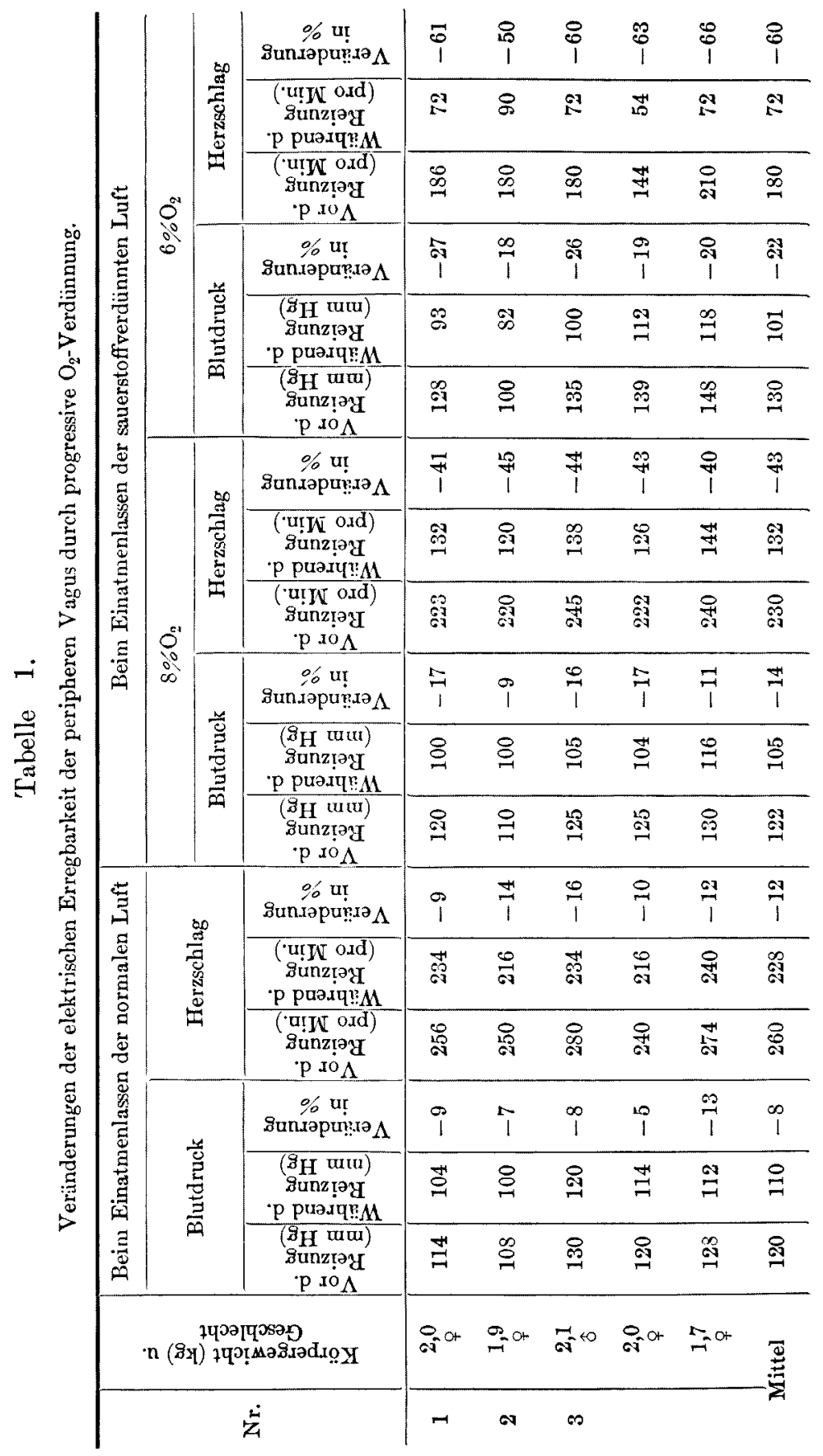


langsam herabgesetzt und kurz vor dem 'Tode, welcher bei ca. $5 \% \mathrm{O}_{2}$ eintrat, belief sich im Durchschnitt der Blutdruckabstieg auf $18 \%$ und die Abnahme der Herzschläge auf 44\%. Diese Veründerungen in einigen Stadien im Verlaufe der $\mathrm{O}_{2}-$ Verdünnung sind in beigefügter Tabelle (Tabelle 1) wiedergegeben.

"Abnahmeprozentsatz des Blutdrucks und der Herzschläge" in der Tabelle bedeutet die prozentuale Abnahme der beiden während der Reizung im Vergleich mit dem Werte vor der Reizung. Die Pulsfrequenz vor und während der Reizung ist pro Minute umgerechnet.

(2) Veränderungen der elektrischen Erregbarkeit des peripheren Sympathikus.

Am Halssympathikus wurden 7 Versuche vorgenommen, welche alle analoge Veränderungen ergaben.

\section{Tabelle 2.}

Verïnderungen der elektrischen Erregbarkeit der peripheren Sympathikus durch progressive $\mathrm{O}_{2}$-Verdinnung.

\begin{tabular}{|c|c|c|c|c|c|c|c|c|c|c|}
\hline \multirow{4}{*}{ Nr. } & \multirow{4}{*}{$\begin{array}{c}\text { Körpergewicht } \\
\text { (kg) u. } \\
\text { Geschlecht }\end{array}$} & \multicolumn{9}{|c|}{ Reizschwelle des Halssympathikus (R. A. in $\mathrm{cm}$ ) } \\
\hline & & \multirow{2}{*}{\multicolumn{3}{|c|}{$\begin{array}{c}\text { Beim } \\
\text { Einatmenlassen } \\
\text { der normalen Luft }\end{array}$}} & \multicolumn{6}{|c|}{$\begin{array}{l}\text { Beim Einatmenlassen } \\
\text { der sauerstoffverdünnten Luft }\end{array}$} \\
\hline & & & & & \multicolumn{3}{|c|}{$90_{0}^{\circ} \mathrm{O}_{2}$} & \multicolumn{3}{|c|}{$5 \% \mathrm{O}_{2}$} \\
\hline & & r. & 1. & 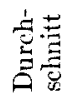 & r. & 1. & 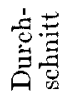 & r. & 1. & 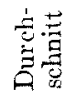 \\
\hline 6 & $\begin{array}{c}1,8 \\
8\end{array}$ & 26 & 22 & 24 & 30 & 28 & 89 & 45 & 40 & 43 \\
\hline 7 & $\begin{array}{c}2,0 \\
\text { 우 }\end{array}$ & 18 & 25 & 22 & 27 & 30 & 29 & 34 & $3 S$ & 36 \\
\hline 8 & $\begin{array}{c}2,2 \\
\text { ơ }\end{array}$ & 27 & 30 & 29 & 30 & 33 & 32 & 47 & 45 & 46 \\
\hline 9 & $\begin{array}{c}2,2 \\
\frac{Q}{3}\end{array}$ & 23 & 26 & 25 & 26 & 32 & 29 & 38 & 47 & 43 \\
\hline 10 & $\begin{array}{c}1,7 \\
9 \\
9\end{array}$ & 25 & 24 & 25 & 25 & 27 & 26 & 32 & 41 & 37 \\
\hline 11 & $\begin{array}{c}2,0 \\
q\end{array}$ & 20 & 23 & 22 & 24 & 30 & 27 & 33 & 42 & 88 \\
\hline 12 & $\begin{array}{c}2,3 \\
\hat{\delta}\end{array}$ & 22 & 25 & 24 & 28 & 30 & 29 & 37 & 40 & 39 \\
\hline \multicolumn{2}{|c|}{ Mittel } & 23 & 25 & 24 & 27 & 30 & 29 & 38 & 42 & 40 \\
\hline
\end{tabular}


Die Reizschwelle des Halssympathikus bei faradischer Roizung lag in normaler Luft bei durchschnittlich $24 \mathrm{~cm}$ R.A., wurde aber ungefähr von der Zeit an, wo der $\mathrm{O}_{2}$-Gehalt der Inspirationsluft $9 \%$ betrug, erniedrigt, d. h. die Erregbarkeit des Nerven gesteigert, und bei ca. $5 \% \mathrm{O}_{2}$ lag die Reizschwelle bei $40 \mathrm{~cm} \mathrm{R.} \mathrm{A.} \mathrm{(R.A.} \mathrm{um} 67 \%$ vergrössert) und hatte damit ihren niedrigsten Wert erreicht; also ist die Erregbarkeit am höchsten gesteigert worden. Danach nahm die Reizschwelle rapid zu, zeigte Neigung, sich ihrem Anfangswerte zu nähern, und lag im Endstadium bei $36 \mathrm{~cm} \mathrm{R.} \mathrm{A.} \mathrm{(eine} \mathrm{Vergrösserung} \mathrm{des} \mathrm{R.} \mathrm{A.} \mathrm{um} \mathrm{50 \% ).} \mathrm{Die} \mathrm{Verände-}$ rungen der Reizschwelle in einigen Stadien des Verlaufes der $\mathrm{O}_{2}$-Verdünnung in den 7 Versuchen sind aus beigefügter Tabelle (Tab. 2) ersichtlich.

(3) Veränderungen der elektrischen Erregbarkeit des peripheren motorischen Nerven.

Zur Ermittlung der Veränderungen der elektrischen Erregbarkeit des peripheren motorischen Nerven nahm ich als Versuchsobjekt den N. peronaeus profundus sinister, und zwar aus dem Grunde, weil das zur Blutgasmessung zu verwendende Blut aus der A. cruralis dextra entnommen und danach ihr peripherer Stumpf unterbunden wird, infolgedessen der

Tabelle 3.

Veränderungen der elektrischen Erregbarkeit des Peronaeus durch progressive $\mathrm{O}_{2}-$ Verdünnung.

\begin{tabular}{|c|c|c|c|c|}
\hline \multirow{3}{*}{$\mathrm{Nr}}$. & \multirow{3}{*}{$\begin{array}{c}\text { Körpergewicht } \\
\text { (kg) u. } \\
\text { Geschlecht }\end{array}$} & \multicolumn{3}{|c|}{ Reizschwelle des Peronaeus (R. A. in $\mathrm{cm}$ ) } \\
\hline & & \multirow{2}{*}{$\begin{array}{l}\text { Beim } \\
\text { Einatmenlassen } \\
\text { der normalen Luft }\end{array}$} & \multicolumn{2}{|c|}{$\begin{array}{l}\text { Bein Einatmenlassen der } \\
\text { sauerstoffverdïnnten Luft }\end{array}$} \\
\hline & & & $9 \% \mathrm{O}_{2}$ & $4 \% \mathrm{O}_{2}$ \\
\hline 13 & $\begin{array}{c}2,2 \\
3\end{array}$ & 37 & 42 & 80 \\
\hline 14 & $\begin{array}{c}1,9 \\
0 \\
0\end{array}$ & 55 & 60 & 95 \\
\hline 15 & $\begin{array}{c}1,6 \\
P\end{array}$ & 45 & 50 & 92 \\
\hline 16 & $\begin{array}{c}2,0 \\
8\end{array}$ & 46 & 50 & 95 \\
\hline 17 & $\begin{array}{c}2,1 \\
q\end{array}$ & 50 & 57 & 93 \\
\hline 18 & $\begin{array}{c}1,8 \\
8\end{array}$ & 43 & 50 & 85 \\
\hline \multicolumn{2}{|c|}{ Mittel } & 46 & 52 & 90 \\
\hline
\end{tabular}


rechte Unterschenkel schon vom Beginn des Versuches an hochgradigen $\mathrm{O}_{2}$-Mangel zeigt.

Bei Durchsicht der Resultate der 6 Versuche findet man fast Übereinstimmendes. Nümlich in gewöhnlicher Luft liegt die Reizschwelle bei durchschnittlich $46 \mathrm{~cm} \mathrm{R.A.,} \mathrm{beginnt} \mathrm{bei} \mathrm{ca.} 9 \% \mathrm{O}_{2}$ langsam sich zu vermindern, d.h. die Erregbarkeit wird allmühlich gesteigert, und schliesslich erreicht die Erregbarkeit bei $4 \% \sigma_{a}$, wo der Tod erfolgt, ihr Maximum, wobei der R. A. im Durchschnitt $90 \mathrm{~cm}$ beträgt, also im Vergleich mit dem Anfangswerte eine Zunahme von 96\% zeigt.

Die Veränderungen der Reizschwelle in einigen hauptsïchlichen Stadien des Verlaufes der $\mathrm{O}_{2}$-Verdünnung zeigt die beigefügte 'Tabelle (Tab. 3).

\section{(4) Veränderungen der vegetativen Funktionen.}

Die obigen Experimente wurden alle mit Anwendung des ,Rebreathers" angestellt. Somit sind die wichtigsten der hierbei entstehenden physiologischen Reaktionen denen in meinen schon mitgeteilten Arbeiten ${ }^{3)}$ gleich. Deshalb will ich sie, um Wiederholungen zu vermeiden, nur der Hauptsache nach im Folgenden schildern.

Was zunächst den Puls betrifft, so begann er kurz nach Beginn des Versuches allmählich sich zu vermehren, erreichte bei ca. $14 \% \mathrm{O}_{2}$ sein Maximum (im Mittel eine Zunahme von $7 \%$ ), nahm danach langsam ab und gelangte bei durchschnittlich $5 \% \mathrm{O}_{2}$ auf sein Minimum (im Mittel eine Abnahme von 60\%); dann beschleunigte er sich wieder und wollte beinahe in Terminalstadium sich seinem Anfangswerte nähern.

Der Blutdruck fing kurz nach Beginn des Versuches zu sinken an, war bei ca. $100 \% \mathrm{O}_{2}$ um 5\%o niedriger als der Anfangswert, stieg danach langsam an, erreichte bei durchschnittlich 5\% $\mathrm{O}_{2}$ sein Maximum (im Mittel ein Anstieg ron $8 \%$ ) und erniedrigte sich irn Endstadium rapid, wobei der Tod erfolgte.

Atemzahl und -tiefe nahmen auch ungeführ von der Zeit an, wo der $\mathrm{O}_{2}$-Gehalt der Inspirationsluft $11 \%$ betrug, allmühlich zu, erreichten bei durchschnittlich $6 \%$ o $\mathrm{O}_{2}$ ihr Maximum (im Durchschnitt war erstere um 59\% und letztere um 170\%ó vermehrt) und verminderte sich im Terminalstadium rasch, um sich dann ihrem Anfungswerte zu nühern.

Die Sauerstoffunsittigung und prozentige Süttigung fingen ungefiihr bei durchschnittlich $16 \% \mathrm{O}_{2}$ allmählich sich zu verändern an, was darin bestand, dass erstere zu- und letztere abnahm, was immer ausgesprochener wurde, bis im Endstadium jene ihr Maximum (eine Zunahme von $670 \%$ ) und diese ihr Minimum (eine Abnahme ron $\$ 9 \%$ ) erreichte.

Auch der $\mathrm{CO}_{2}$-Gehalt des arteriellen Blutes fing fast zugleich mit der Atemzahl und -tiefe sich zu verïndern an, verminderte sich mit zunehmender $\mathrm{O}_{2}$-Verdïnnung immer mehr, um im Terminalstadium sein Minimum (im Durchschnitt eine Abnahme von $52 \%$ ) zu erreichen.

Die Sauerstoffaufnahme pro Minute betrug bis zu der Zeit, wo der $\mathrm{O}_{2}$-Gehalt $16 \%$ erreichte, im Mittel $23 \mathrm{ccm}$, nahm danach mit steigender Sauerstoffverdünnung allmühlich 
ab, um im Endstadium ihr Minimum zu erreichen, wobei sie $7 \mathrm{~cm}$ (eine Abnahme von $69 \%$ in Vergleich mit dem Anfangswerte) zählte.

Die Veründerungen des Blutgasgehaltes und der $\mathrm{O}_{2}$-Aufnahme in einigen hauptsächlichen Stadien des Verlaufes der Untersuchung sind aus Tabelle 4 ersichtlich.

Es betrug im Durchschnitt die Lebensdauer 2 Stunden 34 Minuten, die Gesamtsauerstoffaufnahme $2410 \mathrm{ccm}$ und die erreichte maximale $\mathrm{O}_{2}$-Verdünnung $4 \%\left(\mathrm{O}_{2}\right.$-Gehalt in Volumprozent).

Tabelle 4.

Veränderungen der regetativen Funktionen durch progressive $\mathrm{O}_{2}$-Verduinnung. (Durchschnitt aller Versuche)

\begin{tabular}{|c|c|c|c|c|c|c|c|c|}
\hline 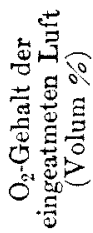 & 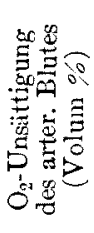 & 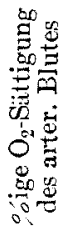 & 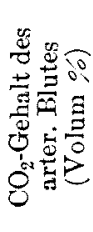 & 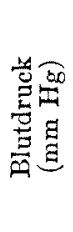 & 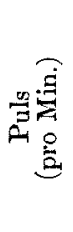 & 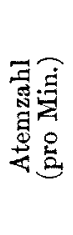 & 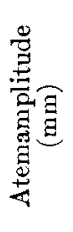 & 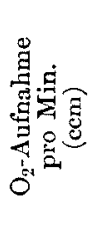 \\
\hline 21 & 2,1 & 89,0 & 48,6 & 116 & 264 & 78 & 3 & - \\
\hline 8 & 9,0 & 51,0 & 34,0 & 120 & 238 & 115 & 5 & 13 \\
\hline 6 & 11,5 & 32,7 & 25,2 & 122 & 158 & 124 & 8 & 7 \\
\hline
\end{tabular}

(II) Ver änderungen der Erregbarkeit der Nerven bei konstanter Sauerstoffverdünnung.

In dieser Versuchsreihe habe ich, wie früher erwähnt, die Tiere aus dem Douglas'schen Sacke Luft, deren $\mathrm{O}_{2}-$ Gehalt auf $7 \%$ vermindert worden war, atmen lassen, wodurch schneller dauernde Anoxämie erzeugt wurde als mit dem „Rebreather", und habe die dadurch veränderte Erregbarkeit der Nerven untersucht.

\section{(1) Veränderungen der elektrischen Erregbarkeit des peripheren Vagus.}

Zuerst untersuchte ich beim Einatmenlassen normaler Luft die Erregbarkeit des cervikalen Vagus und daran anschliessend seine Veränderungen beim Einatmenlassen verdünnter Luft $\left(7 \% \mathrm{O}_{2}\right)$. 5 Versuche darüber ergaben fast analoge Resultate.

Wie aus Tabelle 5 ersichtlich, betrug beim Atmen in normaler Luft im Durchschnitt die Blutdrucksenkung resp. die Herzschlagabnahme, welche durch zehn Sekunden lange Reizung mit Induktionsströmen bei einem R. A. von $20 \mathrm{~cm}$ herbeigeführt wurde $10 \%$ resp. 13\%. Als man dann die Trachealkanüle mit dem sauerstoffarme Luft enthaltenden Dou$\mathrm{gl}$ a s'schen Sacke verband, da zeigten sie bald danach infolge plötzlich eingetretenen $\mathrm{O}_{2}$ - 
Mangels Unruhe, betrüchtlich schwankende Atemzahl und -tiefe, unregelmässigen Puls und Blutdruck, somit schwankenden Gasgehalt des arteriellen Blutes. Aber wie das auch von $\mathrm{D}_{0} \mathrm{i}^{\text {t) }}$ nachgewiesen wurde, trat ungef:ilhr 15 Minuten nach Beginn der Einatmung der verdünnten Luft der Kompensationsmechanismus des Organismus gegenüber Sauerstoffmangel allmählich zutage, sodass die Werte der Blutgase gewisse Zeit lang ziemlich konstant wurden.

Folglich begann ich die Versuche frühestens 15 Minuten nach Beginn des Einatmenlassens verdünnter Luft, setzte es ungefähr 40 Minuten lang fort und ermittelte zwischendurch alle zehn bis fünfzehn Minuten, im ganzen dreimal, die Erregbarkeit für gleich starke Reize, um die zeitlichen Schwan-

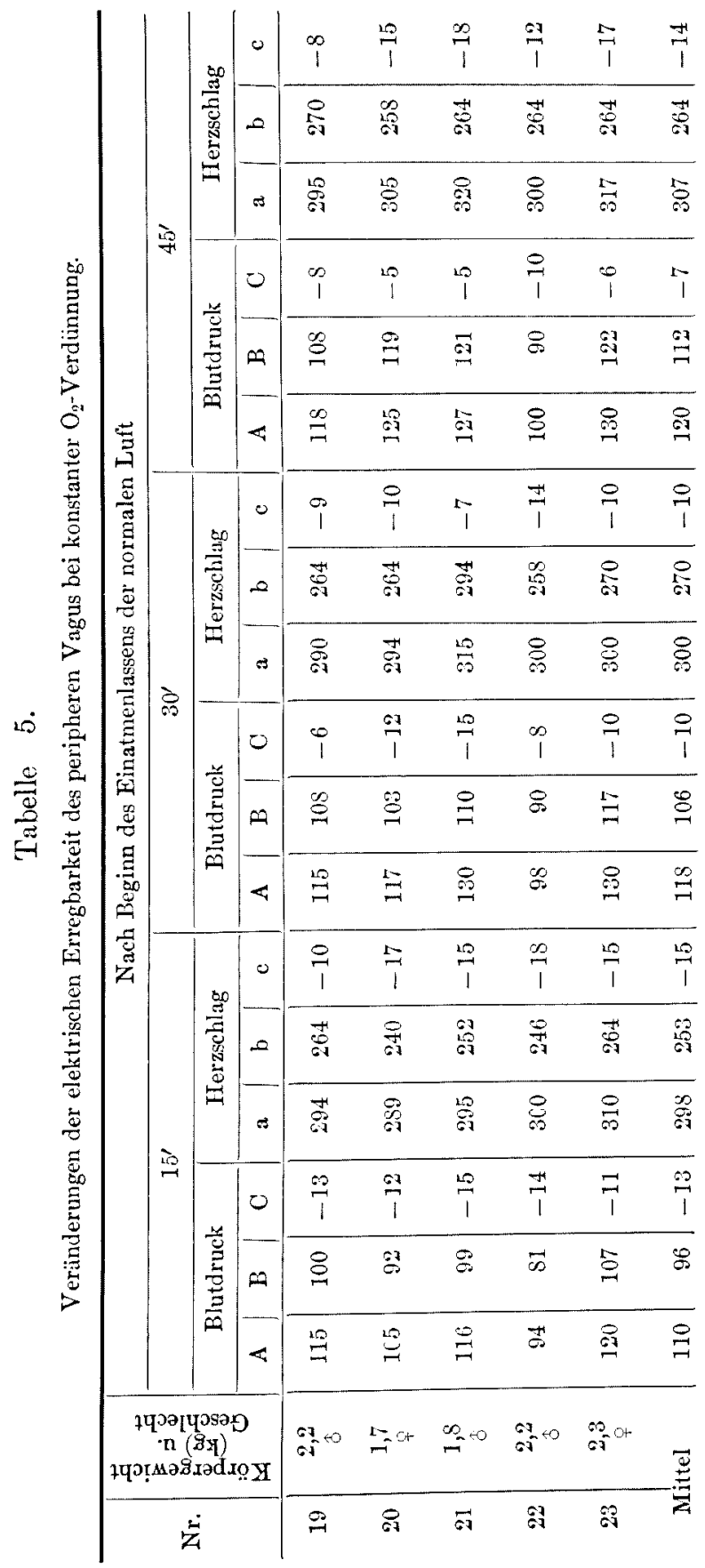




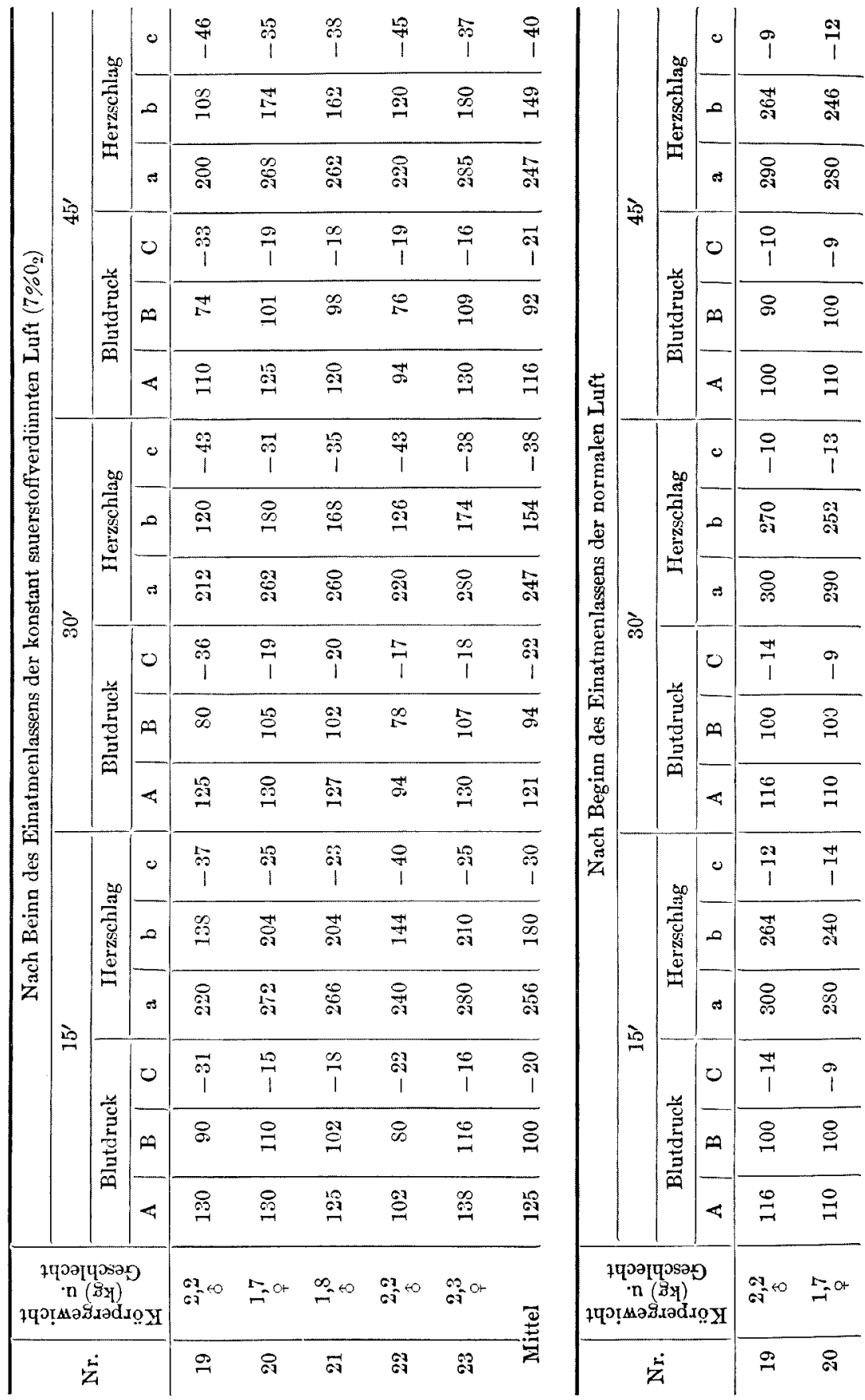




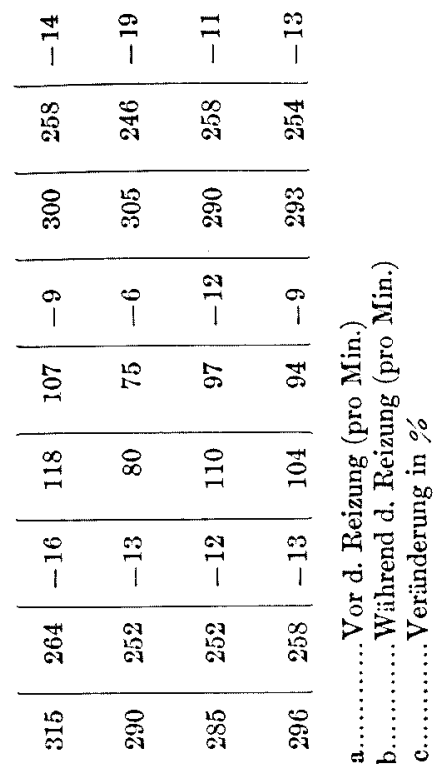

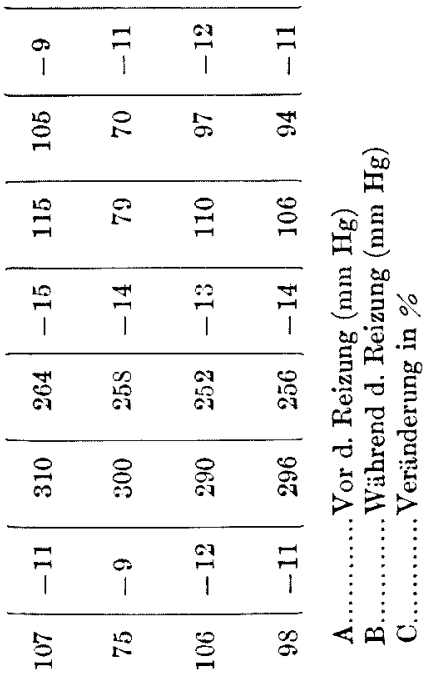

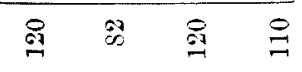

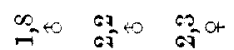$$
\text { क }
$$

kungen der Erregbarkeit bei Anoxämie zu untersuchen. Es zeigte sich indessen, dass die Erregbarkeit, wie aus Tab. 5 ersichtlich, durch eine gewisse Anoxämie in bestimmter Weise beeinflusst wurde, aber keine merklichen Veränderungen zeigte. Lässt man verdünnte Luft, deren Sauerstoffgehalt 7\% betrügt, einatmen, so erfährt der Blutdruck durch Vagusreize, wie die oben erwähnten, in Mittel eine Erniedrigung von $21 \%$, also eine Zunahme von durchschnittlich 110\% im Vergleich mit der Druckherabsetzung in gewöhnlicher Luft. Ferner verlangsamten sich die Herzschläge im Mittel um 36\%, was einer Verstärkung der Pulsabnahme von durchschnittlich $177 \%$ im Vergleich mit der Verlangsamung in normaler Luft entspricht. Derartige Erscheinungen sprechen offenbar dafür, dass hierbei durch Anoxämie der periphere Vagus in seiner elektrischen Erregbarkeit gesteigert wird.

Nach dieser Untersuchung in verdünnter Luft liess ich die Tiere wieder normale Luft einatmen und begann ca. 15 Minuten danach, nachdem der Blutgasgehalt zur Norm zurückgekehrt war, wieder die Erregbarkeit des Nerven zu prüfen, was wiederum die Werte vor dem Einatmenlassen verdünnter Luft ergab.

(2) Veränderungen der elektrischen Erregbarkeit des peripheren Sympathikus.

Nach derselben Methode, wie beim Vagus, habe ich auch beim Hals- 


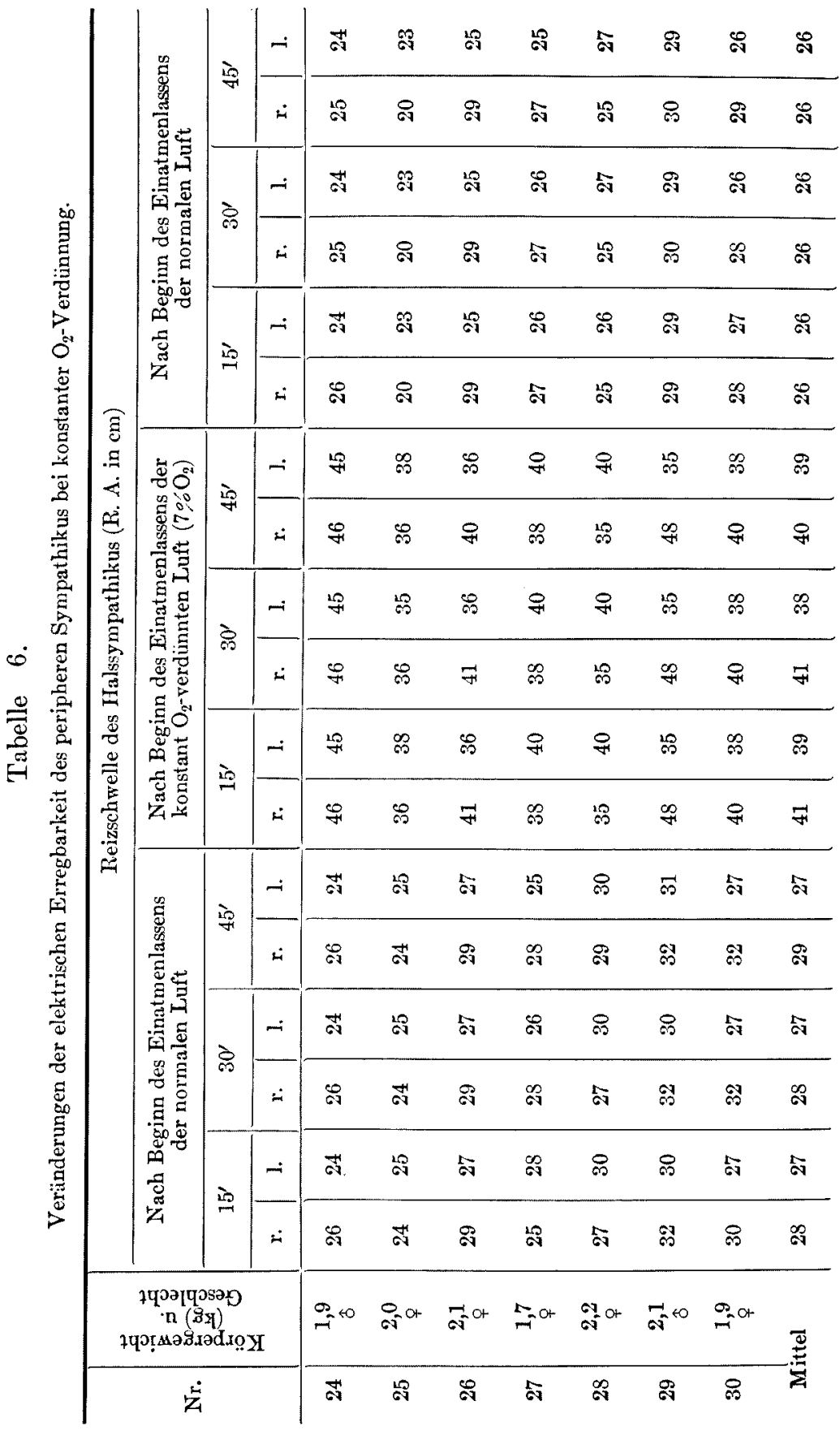


sympathikus die Veränderungen der elektrischen Erregbarkeit untersucht und, wie Tab. 6 veranschaulicht, in 7 Versuchen gefunden, dass der Sch wellenreiz dieses Nerven bei der Atmung in normaler Luft im Mittel bei 28 $\mathrm{cm}$ R. A. lag, aber 15 Minuten nach der Atmung in verdünnter Luft auf eine Stromstärke bei durchschnittlich $40 \mathrm{~cm}$ R. A. (eine Zunahme des R. A. von $43 \%$ ) herabgesetzt wurde, dass also die Erregbarkeit deutlich gesteigert wurde. Dann liess ich wieder gewöhnliche Luft einatmen und, nachdem die Anoxämie zurückgegangen war, untersuchte ich wieder die Erregbarkeit des Nerven im Normalzustand, was ergab, dass sie, die in verdünnter Luft erhöht war, zur Norm zurückgekehrt war.

(3) Veränderungen der elektrischen Erregbarkeit des motorischen Nerven.

In 5 Versuchen wurde die Erregbarkeit des N. peronaeus profundus gemessen (Tab. 7 ). Der Schwellenreiz dieses Nerven lag beim Atmenlassen normaler Luft durchschnittlich bei $47 \mathrm{~cm} \mathrm{R.A.,} \mathrm{verminderte} \mathrm{sich} \mathrm{aber}$ während der Einatmung verdünnter Luft mit 7\%,o $\mathrm{O}_{2}$ auf eine Stromstïrke

Tabelle 7.

Verïderungen der elektrischen Erregbarkeit des Peronaeus bei konstanter $\mathrm{O}_{2}$-Verduinnung.

\begin{tabular}{|c|c|c|c|c|c|c|c|c|c|c|}
\hline \multirow{3}{*}{ Nr. } & \multirow{3}{*}{$\begin{array}{c}\text { Körpergewicht } \\
\text { (kg)u. } \\
\text { Geschlecht }\end{array}$} & \multicolumn{9}{|c|}{ Reizschwelle des Peronaeus (R. A. in $\mathrm{cm}$ ) } \\
\hline & & \multicolumn{3}{|c|}{$\begin{array}{l}\text { Nach Beginn des } \\
\text { Einatmenlassens } \\
\text { der normalen Luft }\end{array}$} & \multicolumn{3}{|c|}{$\begin{array}{c}\text { Nach Beginn des } \\
\text { Einatmenlassens } \\
\text { der konstant } \\
\text { Or-verdünnten } \\
\text { Luft }\left(7960_{2}\right)\end{array}$} & \multicolumn{3}{|c|}{$\begin{array}{l}\text { Nach Beginn des } \\
\text { Einatmenlassens } \\
\text { der normalen Luft }\end{array}$} \\
\hline & & $18^{\prime}$ & $33^{\prime}$ & $48^{\prime}$ & $18^{\prime}$ & $83^{\prime}$ & $4 S^{\prime}$ & $18^{\prime}$ & $33^{\prime}$ & $4 S^{\prime}$ \\
\hline 19 & $\begin{array}{c}2,2 \\
0\end{array}$ & 45 & 45 & 45 & 60 & 60 & 60 & 42 & 42 & 42 \\
\hline 20 & $\frac{1,7}{7}$ & 55 & 55 & 55 & 72 & 72 & 72 & 50 & 51 & 50 \\
\hline 21 & $\begin{array}{c}1,8 \\
\hat{3}\end{array}$ & 46 & 46 & 46 & 60 & 60 & 61 & 45 & 45 & 45 \\
\hline 22 & $\begin{array}{c}2,2 \\
\hat{\delta}\end{array}$ & 47 & 47 & 46 & 63 & 64 & 64 & 47 & 46 & 46 \\
\hline 23 & $\begin{array}{c}2,3 \\
7\end{array}$ & 42 & 42 & 42 & 57 & 56 & 57 & 40 & 41 & 40 \\
\hline & ttel & 47 & 47 & 47 & 62 & 62 & 63 & 45 & 45 & 45 \\
\hline
\end{tabular}


bei durchschnittlich $62 \mathrm{~cm} \mathrm{R.A.} \mathrm{(eine} \mathrm{Vergrösserung} \mathrm{des} \mathrm{R.} \mathrm{A.} \mathrm{von} \mathrm{durch-}$ schnittlich $32 \%$ ), was zeigt, dass die Erregbarkeit gesteigert ist. Dann kehrte er durch Atmen in normaler Luft wieder zur Norm zurück.

(4) Veründerungen der vegetativen Funktionen.

Die Versuche, in denen ich aus dem Dougl a s'schen Sacke dauernd sauerstoffarme Luft einatmen liess, ergaben, dass die Veränderungen der Atmung, des Kreislaufes, der Gase des arteriellen Blutes und der Sauerstoffaufnahme pro Minute in allen Füllen nicht erheblich voneinander abwichen. Sie werden der Bequemlichkeit halber in Durscbnittszahlen in Tab. 8 zusammengefasst angegeben.

\section{Tabelle 8 .}

Verïnderungen der vegetativen Funktionen durch die konstante $\mathrm{O}_{2}$-Verdünnung. (Durchschnitt aller Versuche)

\begin{tabular}{|c|c|c|c|c|c|c|c|c|}
\hline 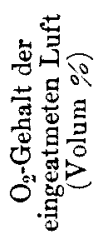 & 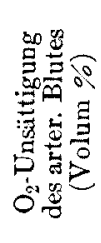 & 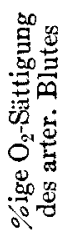 & 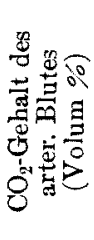 & 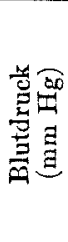 & 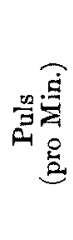 & 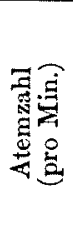 & 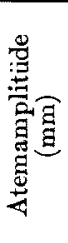 & 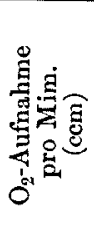 \\
\hline $\begin{array}{r}21 \\
7\end{array}$ & $\begin{array}{l}2,0 \\
9,3\end{array}$ & $\begin{array}{l}89,5 \\
50,2\end{array}$ & $\begin{array}{l}49,4 \\
38,2\end{array}$ & $\begin{array}{l}110 \\
114\end{array}$ & $\begin{array}{l}280 \\
242\end{array}$ & $\begin{array}{l}66 \\
79\end{array}$ & $\begin{array}{l}3 \\
6\end{array}$ & $\begin{array}{l}27 \\
19\end{array}$ \\
\hline
\end{tabular}

Kontrollversuche.

Un zu ermitteln, ob die Erregbarkeit der peripheren Nerven durch Faktoren ausser der Anoxämie, z. B. Blutentnahme u. ä., oder auch während der Versuchsdaner spontan verändert wird oder nicht, habe ich statt Einatmenlassens von Luft von konstanter oder allmählich zunehmender Sauerstoffarmut für fast gleich lange Zeit, wie in den vorigen Versuchen, gewöhnliche Luft inspirieren lassen und so nach gleichen Verfahren wie früher die Erregbarkeit der oben geprüften drei Nerven gemessen, und zwar in je zwei Versuchen für je eine Nervenart. Es ergaben sich aber keine besonderen Veränderungen der Erregbarkeit der peripheren Nerven während der ganzen Versuchsdauer.

\section{Zusammenfassung.}

Die Ergebnisse der Untersuchungen lassen sich, wie folgt, kurz zusammenfassen : Wenn die $\mathrm{O}_{2}$-Verdünnung durch den ,Rebreather" all- 
mählich gesteigert wird, fängt die elektrische Erregbarkeit des Halsvagus, -sympathikus und peripheren motorischen Nerven ungefïhr bei $8-9 \% \mathrm{O}_{2-}$ Gehalt der Einatmungsluft sich allmühlich zu erhöhen an und erreicht bei allen bei 5-6\% $\mathrm{O}_{2}$ ihr Maximum, wobei der Halsvagus die am meisten gesteigerte Erregbarkeit erkennen lässt, der motorische Nerv an zweiter und der Halssympathikus an dritter Stelle steht. Der Vagus und Sympathikus werden ungefähr zu der Zeit, wo infolge immer steigender Anoxämie der Tod droht, in der Regel wieder in ihrer Erregbarkeit herabgesetzt und zeigen Neigung, sich ihrem Anfangswerte zu nähern. Dies beruht wohl auf nichts anderem als auf Ermüdungserscheinungen der Nerven, welche durch einen über eine bestimute Stärke gesteigerten $\mathrm{O}_{2}$-Mangel bedingt sind. Es zeigt anderseits aber der periphere motorische Nerv offenbar keine derartigen Phänomene, sondern er behält seine gesteigerte Erregbarkeit bis zum Exitus, was wohl darauf hinweist, dass dieser Nerv eine relativ starke Toleranz gegen Anoxämie hat.

Was die Variationen des Pulses und Blutdruckes im Verlaufe der erwähnten Versuche anbelangt, so macht sich immer bei gesteigerter $\mathrm{O}_{2}$-Verdünnung Blutdruckanstieg resp. Pulsabnahme geltend, von denen ersterer resp. letztere wohl hauptsächlich auf die Erregung des vasomotorischen resp. Vaguszentrums zurückzuführen ist, wie dies bei Asphyxie der Fall ist. Aber man muss, wie meine Untersuchungen zeigen, auch die dabei auftretenden Veränderungen der Erregung der peripheren vegetativen Nerven in Erwïgung ziehen.

Dann ist zu erwähnen, dass auch in dem Falle, wo durch Einatmenlassen konstant sauerstoffarmer $\left(7 \% 0 \mathrm{O}_{2}\right)$ Luft aus dem Douglas'schen Sacke ziemlich rasch starke und dauernde Anoxïmie erzeugt wurde, die peripheren Nerven in ihrer elektrischen Erregbarkeit gleiche Veränderungen wie die oben erwähnten zeigen, welche fast gleich stark sind wie die im Stadium mit ca. $7 \% \mathrm{O}_{2}$ bei progressiver $\mathrm{O}_{2}$-Verdünuung. Auch ist hierbei die prozentuale Steigerung der elektrischen Erregbarkeit beim Vagus am grössten, beim Sympathikus am zweitgrössten und beim motorischen Nerven am kleinsten.

\section{Schluss.}

Durch progressive Sauerstoffverdünnung steigert sich allmählich die elektrische Erregbarkeit des peripheren Vagus und Sympathikus, erreicht bei ca. 5-6\% $\mathrm{O}_{2}$ ihr Maximum und füngt bei noch zunehmender $\mathrm{O}_{2}$-Verdünnung und herannahendem Tode abzunehmen an, geht aber nicht unter 
den Anfangswert herunter.

Auch beim peripheren motorischen Nerven wird die Erregbarkeit desto mehr erhöht, je mehr die $\mathrm{O}_{-}-$Verdünnung verstärkt wird, und wird bei durchschnittlich $4 \% \mathrm{O}_{2}$ maximal, welche Vermehrung bis zum Tode andauert.

Auch bei konstanter $\mathrm{O}_{2}$-Verdünnung $\left(\mathrm{O}_{2}\right.$-Gehalt $\left.7 \%\right)$ werden der periphere Vagus, Sympathikus und motorische Nerv in ihrer Erregbarkeit auf gleiche Weise gesteigert. 